\title{
Radial Infall onto a Massive Molecular Filament
}

\author{
Cara Battersby $^{1}$, Philip C. Myers ${ }^{1}$, Yancy L. Shirley ${ }^{2}$, Eric Keto ${ }^{1}$ \\ and Helen Kirk ${ }^{3}$ \\ ${ }^{1}$ Harvard-Smithsonian Center for Astrophysics 60 Garden St., Cambridge, MA 02138 USA \\ email: cbattersby@cfa.harvard.edu \\ ${ }^{2}$ Steward Observatory, 933 North Cherry Avenue, Tucson, AZ 85721, USA \\ ${ }^{3}$ Origins Institute, McMaster University, Hamilton, ON, L8S 4M1, Canada
}

\begin{abstract}
The newly discovered Massive Molecular Filament (MMF) G32.02+0.05 ( 70 pc long, $10^{5} \mathrm{M}_{\odot}$ ) has been shaped and compressed by older generations of massive stars. The similarity of this filament in physical structure (density profile, temperature) to much smaller star-forming filaments, suggests that the mechanism to form such filaments may be a universal process. The densest portion of the filament, apparent as an Infrared Dark Cloud (IRDC) shows a range of massive star formation signatures throughout. We investigate the kinematics in this filament and find widespread inverse P cygni asymmetric line profiles. These line asymmetries are interpreted as a signature of large-scale radial collapse. Using line asymmetries observed with optically thick $\mathrm{HCO}^{+}(1-0)$ and optically thin $\mathrm{H}^{13} \mathrm{CO}^{+}(1-0)$ across a range of massive star forming regions in the filament, we estimate the global radial infall rate of the filament to range from a few 100 to a few $1000 \mathrm{M}_{\odot} \mathrm{Myr}^{-1} \mathrm{pc}^{-1}$. At its current infall rate the densest portions of the cloud will more than double their current mass within a Myr.
\end{abstract}

Keywords. stars: formation, ISM: kinematics and dynamics

\section{Data and Modelling}

We observed 19 pointings toward the highest infrared-extinction region of the G32.02 + 0.05 (G32) Massive Molecular Filament (MMF; see Figure 1) with the Arizona Radio Observatory $12 \mathrm{~m}$ over 2 nights in March 2013. We used the MAC backend in 2 IF mode with $0.08 \mathrm{~km} \mathrm{~s}^{-1}$ resolution to observe the $\mathrm{HCO}^{+}$and $\mathrm{H}^{13} \mathrm{CO}^{+}$(1-0) lines. We used Saturn for main beam calibration, observed in position switching mode, and reduced the data using GILDAS CLASS $\dagger$ software. Our effective spatial resolution is $\sim 70^{\prime \prime}$, or 1.9 pc at the distance of G32 (5.5 kpc; Battersby et al. 2014).

We model the spectral line emission by implementing a physical model of the G32 filament based on Herschel data into the radiative transfer code MOLLIE (Keto \& Rybicki 2010). We create $\mathrm{N}\left(\mathrm{H}_{2}\right)$ and $\mathrm{T}_{\text {dust }}$ maps of the filament with data from Hi-GAL (Molinari et al. 2010) using the methods described in Battersby et al. (2011). The average temperature profile across the filament (a cut perpendicular to the long axis of the filament) is relatively flat, with an average $\mathrm{T}_{d u s t}$ of $16 \mathrm{~K}$, with a small decrease toward the center of the filament. The average $\mathrm{N}\left(\mathrm{H}_{2}\right)$ profile across the filament can be fit with a Plummer or Gaussian profile. A Plummer profile has the form of $\rho(r)=\rho_{0} /\left[1+\left(r / r_{\text {flat }}\right)^{2}\right]^{p / 2}$ and our best fit value for the inner flat radius, $\mathrm{r}_{\text {flat }}$, is $0.5 \mathrm{pc}$ and the power-law index, $\mathrm{p}$, is 2.1. Our best Gaussian fit gave a width (FWHM) of 2.2 pc. The Plummer profile index is very similar to that found in Arzoumanian et al. (2011), yet our inner flat region is 15 times larger. 


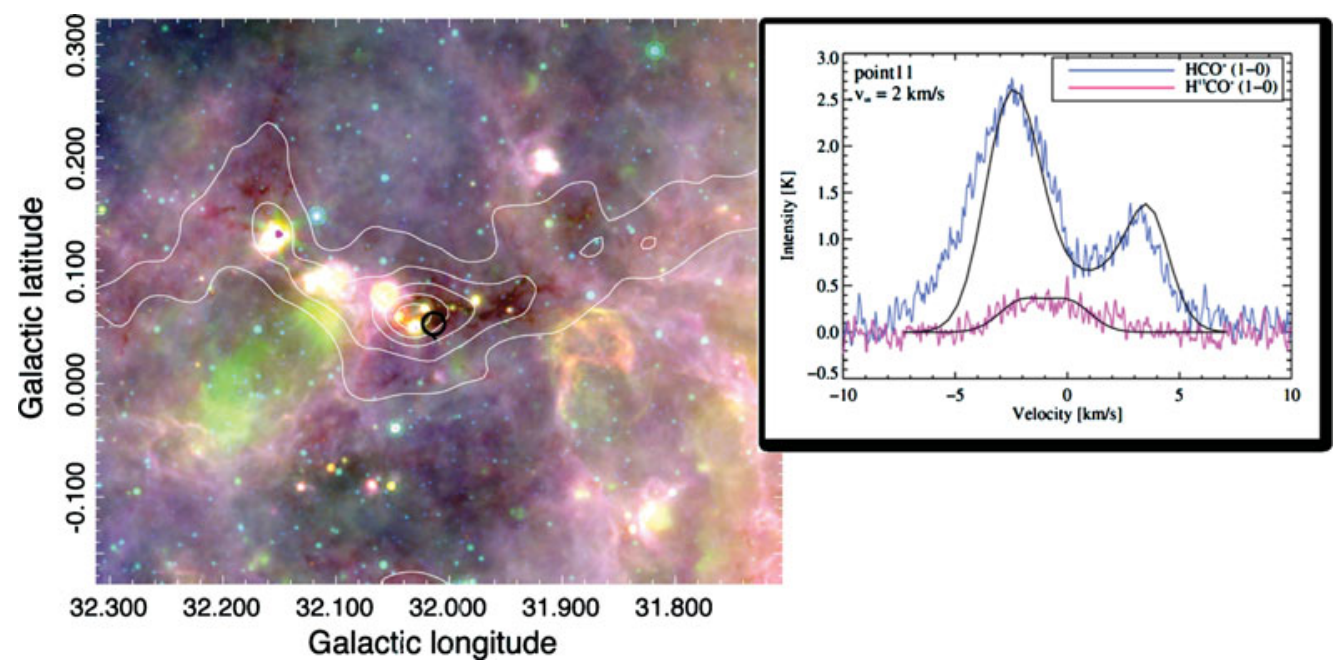

Figure 1. The G32.02+0.05 Massive Molecular Filament (MMF) shown on the left with 70 $\mu \mathrm{m}$ (Molinari et al. 2010) in red, $24 \mu \mathrm{m}$ (Carey et al. 2009) in green, and $8 \mu \mathrm{m}$ (Benjamin et al. $2003)$ in blue. The white contours show ${ }^{13} \mathrm{CO}$-derived column density contours $\left(\mathrm{N}\left(\mathrm{H}_{2}\right)=0.5-2.5\right.$ $\times 10^{22} \mathrm{~cm}^{-2}$ ) from the Galactic Ring Survey (Jackson et al. 2006). The filament extends over 70 pc with a total mass of $\sim 10^{5} \mathrm{M}_{\odot}$ (Battersby et al. 2014). The spectra on the right is $\mathrm{HCO}^{+}$and $\mathrm{H}^{13} \mathrm{CO}^{+}$(1-0) emission from the Arizona Radio Observatory $12 \mathrm{~m}$. The black line shows the MOLLIE radiative transfer model spectra for the filament with an infall speed of $2 \mathrm{~km} \mathrm{~s}^{-1}$.

We create a physical model of a cylindrical filament using the average temperature and best-fit Plummer density profile and implement this into the radiative transfer code MOLLIE (Keto \& Rybicki 2010). We add a variety of different uniform infall speeds to the physical model and run the radiative transfer code to produce simulated $\mathrm{HCO}^{+}$and $\mathrm{H}^{13} \mathrm{CO}^{+}$(1-0) spectra. We compare these to our observations (see Figure 1) and find that an infall speed of $2 \mathrm{~km} \mathrm{~s}^{-1}$ best fits the data.

\section{Results}

We detect widespread infall signatures (inverse P cygni profiles) toward the G32 massive molecular filament. Herschel data show that the G32 filament density profile is well-modeled by a Gaussian or Plummer profile with an index of 2, while the temperature profile is mostly flat with a small dip in the center. We implement a physical model of the filament into a radiative transfer code and find that the inverse $\mathrm{P}$ cygni profile is best fit with an infall velocity of $2 \mathrm{~km} \mathrm{~s}^{-1}$. Using a simple equation for the mass flow across a cylindrical surface, $\dot{M}=\rho \sigma v$, we find that at a radius of $1 \mathrm{pc}$, and a variety of reasonable densities from our Herschel fits, an infall speed of $2 \mathrm{~km} \mathrm{~s}^{-1}$ corresponds to several 100 to several $1000 \mathrm{M}_{\odot} \mathrm{pc}^{-1} \mathrm{Myr}^{-1}$. The mass per unit length of the G32 filament varies from several 100 to several $1000 \mathrm{M}_{\odot} \mathrm{pc}^{-1}$, so our infall rates correspond to roughly doubling the mass of the filament on the timescale of a Myr.

\section{References}

Arzoumanian, D., Andre, P., Didelon, P., et al. 2011, A\&A, 529, L6

Battersby, C., Bally, J., Ginsburg, A., et al. 2011, A\&AA, 535, A128

Battersby, C., Ginsburg, A., Bally, J., et al. 2014, ApJ, 787, 113

Benjamin, R. A., Churchwell, E., Babler, B. L., et al. 2003, PASP, 115, 953 
Carey, S. J., Noriega-Crespo, A., Mizuno, D. R., et al. 2009, PASP, 121, 76

Jackson, J. M., Rathborne, J. M., Shah, R. Y., et al. 2006, ApJS, 163, 145

Keto, E. \& Rybicki, G. 2010, ApJ, 716, 1315

Molinari, S., Swinyard, B., Bally, J., et al. 2010, A\&\&A, 518, L100

Molinari, S., Bally, J., Noriega-Crespo, A., et al. 2011, ApJL, 735, L33 